\title{
Polymer optical fiber Bragg grating acting as an intrinsic biochemical concentration sensor
}

\author{
Wei Zhang, ${ }^{1, *}$ David Webb, ${ }^{1}$ and Gangding Peng ${ }^{2}$ \\ ${ }^{1}$ Photonics Research Group, Aston University, Birmingham B4 7ET, UK \\ ${ }^{2}$ School of Electrical Engineering and Telecommunications, University of New South Wales, Sydney, NSW 2052, Australia \\ *Corresponding author: w.zhang@aston.ac.uk
}

Received January 19, 2012; revised February 22, 2012; accepted February 22, 2012;

posted February 22, 2012 (Doc. ID 161768); published April 12, 2012

\begin{abstract}
We demonstrate an intrinsic biochemical concentration sensor based on a polymer optical fiber Bragg grating. The water content absorbed by the polymer fiber from a surrounding solution depends on the concentration of the solution because of the osmotic effect. The variation of water content in the fiber causes a change in the fiber dimensions and a variation in refractive index and, therefore, a shift in the Bragg wavelength. Saline solutions with concentration from $0 \%$ to $22 \%$ were used to demonstrate the sensing principle, resulting in a total wavelength shift of $0.9 \mathrm{~nm}$, allowing high-resolution concentration measurements to be realized. (c) 2012 Optical Society of America

OCIS codes: $\quad 060.2370,250.5460$.
\end{abstract}

Fiber Bragg grating (FBG) sensors inscribed in silica optical fiber have become an increasingly mature sensing technology, especially for strain and temperature monitoring [1]. FBGs have also been used for biochemical sensing, where the usual aim is to produce a signal that is ideally proportional to the concentration of a chemical or biochemical species. However, silica is itself quite inert, and it is consequently necessary to add additional fiber coatings to provide the necessary biochemical sensitivity [2]. More recently, FBG sensors have been inscribed into poly(methyl methacrylate) (PMMA) based plastic optical fiber (POF) in both step-index [3] and microstructured geometries [4]. The physical and chemical properties of polymeric materials are rather different from those of silica and may offer advantages in certain situations.

In this paper, a new type of fiber-optic biochemical concentration sensor based on a polymer optical fiber Bragg grating (POFBG) is described. The fiber swells or shrinks as a function of analyte concentration, which changes the wavelength of a Bragg grating inscribed in the fiber. The feasibility of this sensing concept is demonstrated by a saline concentration sensor. Because this sensing concept does not require excitation of an indicator and can be implemented at any wavelength, it can potentially be exploited to make rugged, inexpensive biochemical sensors for in situ measurement.

The experimental setup is shown in Fig. 1. The POFBG was fabricated by illuminating from above a phase mask of period $1.034 \mu \mathrm{m}$ placed on top of a PMMA-based POF using $325 \mathrm{~nm}$ light from a HeCd laser, which produced a Bragg wavelength of around $1533 \mathrm{~nm}$ [4]. The grating was then attached to a single-mode silica fiber downlead using UV-curable glue. In the experiment, the POFBG was inserted into test tubes of saline solution with different concentrations. The reflection of the POF grating was monitored by using an Ibsen I-MON 400 wavelength interrogation system. The captured grating reflection spectrum is shown in the inset. From it one can see that there are three reflected peaks, indicating a few-mode operation for this step-indexed POF. In the experiment. only the fundamental mode (strongest) was monitored, while the environmental humidity was kept at $\sim 45 \%$ and the temperature at $21{ }^{\circ} \mathrm{C}$.
The POFBG was first inserted into a test tube of distilled water for around $2 \mathrm{~h}$, so that the POFBG was fully swelled by the water, and then pulled out and inserted into a test tube of saline solution. Four samples of saline solution $(7.5 \%, 13 \%, 17.5 \%$, and $22 \%)$ were tested in order of ascending concentration and then in order of descending concentration. The POFBG device was left for $60 \mathrm{~min}$ in each sample to allow equilibrium to be reached. The POFBG wavelength was monitored with an interval of $60 \mathrm{~s}$. A typical captured response is shown in Fig. 2.

From the captured response, one can see that the POFBG wavelength varies significantly as a function of saline concentration. The POFBG wavelength decreases with increased saline concentration, and the process is reversible.

It is well known that PMMA and many other polymers tend to absorb water $[5,6]$. Many polymers exhibit water absorption processes in which clustering of water occurs within the polymer matrix, leading to an absorption increase at higher humidities. This leads to a swelling of the fiber and an increase of index, both of which contribute to an increase in the Bragg wavelength of an FBG written in the fiber. This is the operating principle for the POFBG humidity sensor [7]. The wavelength of the polymer fiber grating can be related to the water content in the polymer fiber. Following [5] and [8], we assume that the Bragg wavelength shifts linearly with water content in the polymer fiber, $W$. Therefore the Bragg wavelength can be expressed as

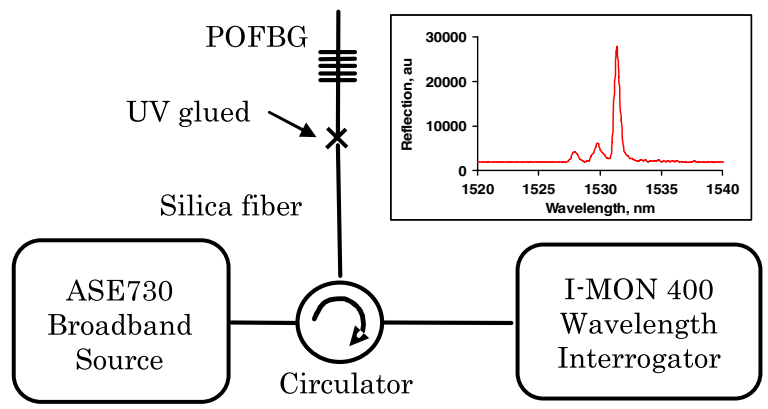

Fig. 1. (Color online) Experimental arrangement. 


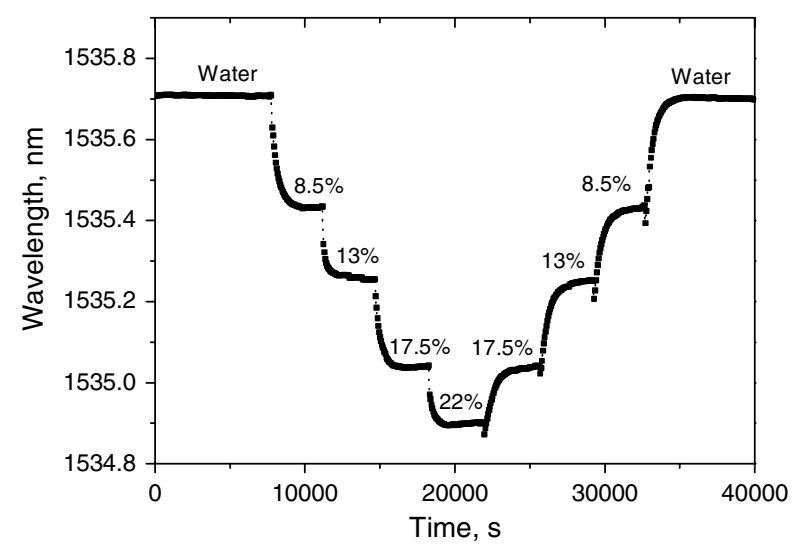

Fig. 2. Measured sensor response against step change of saline concentration.

$$
\lambda_{B}=\lambda_{B, 0}+\alpha W
$$

where $\lambda_{B, 0}$ is the initial Bragg wavelength and $\alpha$ is a coefficient associated with PMMA swelling, water dependence of the refractive index, and swelling-induced stress. The process of water absorption or desorption in PMMA can be described by the diffusion theory of mass transfer [9].

When the polymer fiber is inserted in water, it is fully swollen, giving rise to a maximum wavelength change of the POFBG [5]. If solute exists in the water, then the water content absorbed in the polymer fiber will change as a result of the process of osmosis [10]. In this work, the polymer fiber acts as a semipermeable membrane, which allows solvent (water in this case) to pass through and blocks the solute (salt in this case). The osmotic pressure generated by a solution with a molar concentration $c$ is simply given by [11]

$$
P_{\mathrm{osm}}=c R T
$$

where $R$ is the gas constant $\left(8.314 \mathrm{~J} \mathrm{~K}^{-1} \mathrm{~mol}^{-1}\right)$ and $T$ is the temperature. The rate of water transport between fiber and surrounding solution can be expressed as

$$
Q=\left(\Delta P-P_{\mathrm{osm}}\right) K_{w} S / d,
$$

where $\Delta P$ is a differential hydraulic pressure generated by the water pressure difference inside and outside the fiber, $K_{w}$ is the membrane permeability coefficient for water, $S$ is the membrane area, and $d$ is the membrane thickness. If the outside water pressure is higher than the inside water pressure, $\Delta P$ is positive, driving the water into the polymer fiber. The differential hydraulic pressure will reduce to a minimum and the fiber will be fully swollen in pure water. When the surrounding solution concentration increases, the osmotic pressure increases correspondingly to drive the water content in the fiber into the surrounding solution, consequently increasing the differential hydraulic pressure. This process will stop once there is a balance between the osmotic pressure and the differential hydraulic pressure. Similarly, when the solution concentration decreases, the osmotic pressure decreases, leading to a dominant differential hydraulic pressure that drives the water into the fiber till a new

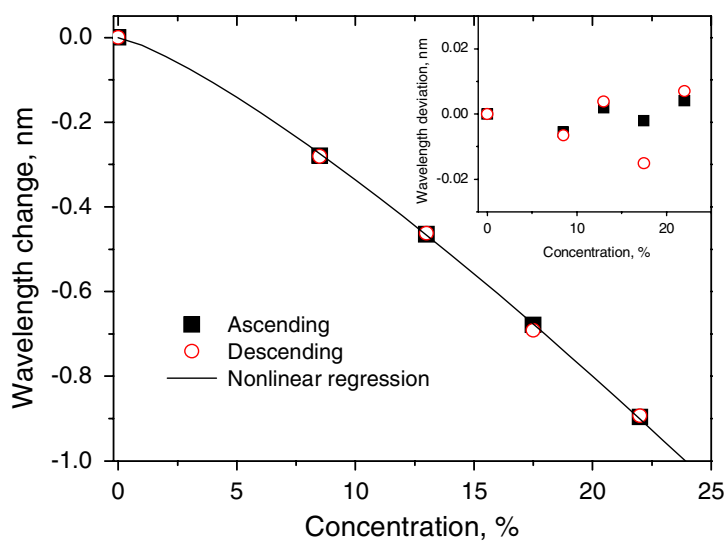

Fig. 3. (Color online) Wavelength change of POFBG versus concentration. Inset: Wavelength deviation for concentration measurement.

pressure balance is established. Therefore, the water content in the polymer fiber depends directly on the solution concentration, which is readily monitored by the wavelength change of the POFBG.

The wavelength change is plotted against concentration in Fig. 3. From this one can see that the POFBG wavelength varies in a nonlinear fashion with the concentration. Similar nonlinearity has been noted in [5,12], where the POFBG wavelength varied nonlinearly with relative humidity. This was attributed to the permeability coefficient for water of many polymeric materials, which varies with relative humidity as a result of the plasticizing effect induced by absorbed water on polymer materials [13]. It leads to a nonlinear change of the water content absorbed in the fiber and thus a nonlinear Bragg wavelength change against relative humidity or relative water pressure.

Nonlinear regression was used to fit the response to an exponential function, the result being shown in Fig. 3. The inset in Fig. 3 shows the wavelength deviation from this regression for each concentration measurement. It can be noted that in the measurements of both increased concentration and decreased concentration, the POFBG wavelength shows almost the same value for the same sample. This performance shows that the sensor is reversible. When the saline concentration varies from $0 \%$ to

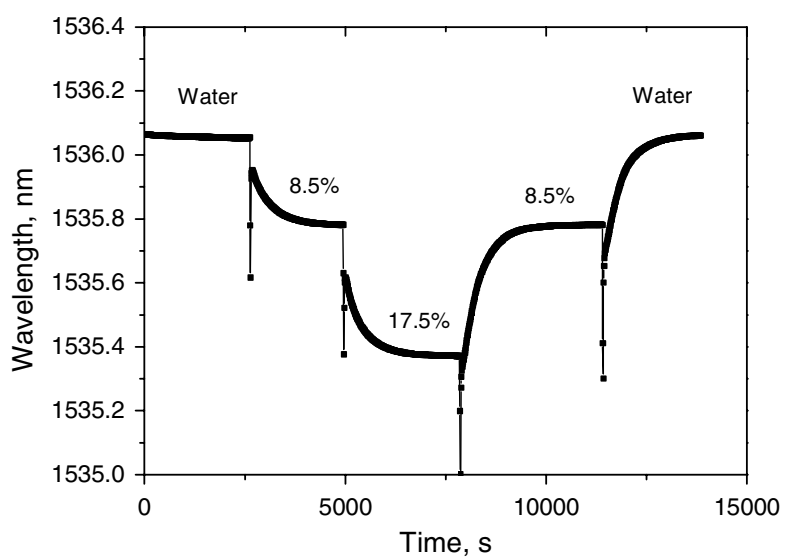

Fig. 4. More detailed sensor response against step change of saline concentration. 


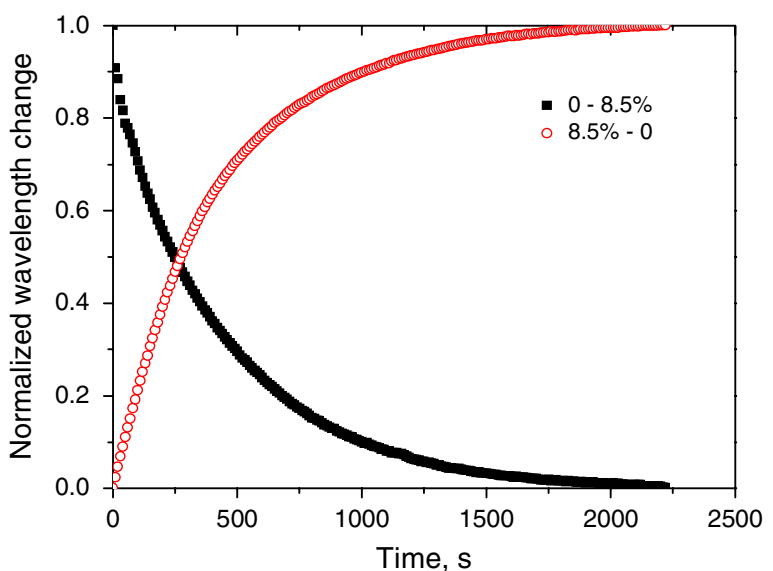

Fig. 5. (Color online) Sensor responses reflecting response time.

$22 \%$, the corresponding wavelength shift of the POFBG sensor is $\sim 0.9 \mathrm{~nm}$. Considering the wavelength resolution of the I-MON 400 wavelength interrogation system (better than $1 \mathrm{pm}$ ), a very accurate measurement of solution concentration can be achieved.

A more detailed time response was captured with an interval of $10 \mathrm{~s}$, as shown in Fig. 4 . It can be seen that there is a large spike in the sensor response during each sample change. This is because when the sensor was pulled out of the solution and exposed to air, the POFBG wavelength dropped sharply as a result of the large negative differential hydraulic pressure between fiber and air, driving water out of the fiber.

It takes some time for the sensor response to reach a stable value. The response to a step concentration change can be approximated by an exponential function, and a response time can be estimated as the time taken to reach $90 \%$ of the equilibrium value. Figure $\underline{5}$ shows the relative wavelength changes for saline concentration changes of $0 \%$ to $8.5 \%$ and $8.5 \%$ to $0 \%$. The estimated response times are 1040 and $960 \mathrm{~s}$, respectively. The response time strongly depends on the diameter and polymer material of the fiber used. For the same type of polymer fiber, it has been demonstrated that a smaller diameter leads to a faster response time [12]. The fiber diameter used in this work is $135 \mu \mathrm{m}$. A further improvement in time response can be expected by use of a grating inscribed in slotted PMMA optical fiber [14], in which water could reach the fiber core in much less time.

The POFBG sensors are sensitive to temperature, although the temperature was kept constant in this work. This cross-sensitivity of the sensor should be considered and eliminated in concentration measurements. This can be realized by using a dual-parameter sensor in which a second, adjacent FBG is inscribed in the silica fiber downlead [7]. Since the silica FBG exhibits no intrinsic humidity/water sensitivity, a well-conditioned response can be achieved.

A biochemical concentration sensor based on a polymer fiber Bragg grating has been proposed and demonstrated in the measurement of saline concentration. Unlike most fiber-optic biosensors, this sensor does not require an immobilized indicator dye; therefore, the performance is not limited by the stability and optical properties of that indicator [2]. The sensor is reversible and thus can be used for in situ repeatable measurement and monitoring of concentration. The grating can be inscribed to provide a response in the near-infrared region to take full advantage of technology developed for fiberoptic communications. The operating principle means that this concept can be used for sensing other singlesolute biochemical solutions. An interesting feature is that the sensing concept is based on the process of osmosis, which is a natural process in many living cells. From this point of view, the device is a real biosensor.

\section{References}

1. R. Willsch, W. Ecke, and H. Bartelt, Proc. SPIE 7753, 775302 (2011).

2. M. E. Bosch, A. J. R. Sánchez, F. S. Rojas, and C. B. Ojeda, Sensors 7, 797 (2007).

3. Z. Xiong, G. D. Peng, B. Wu, and P. L. Chu, IEEE Photon. Technol. Lett. 11, 352 (1999).

4. H. Dobb, D. J. Webb, K. Kalli, A. Argyros, M. C. J. Large, and M. A. van Eijkelenborg, Opt. Lett. 30, 3296 (2005).

5. N. G. Harbach, "Fiber Bragg gratings in polymer optical fibers," Ph.D. dissertation (Ecole Polytechnique Fédérale de Lausanne, 2008).

6. J. A. Barrie and B. Platt, Polymer 4, 303 (1963).

7. C. Zhang, W. Zhang, D. J. Webb, and G.-D. Peng, Electron. Lett. 46, 643 (2010).

8. A. M. Thomas, J. Appl. Chem. 1, 141 (1951).

9. J. Crank, The Mathematics of Diffusion, 2nd ed. (Clarendon, 1975).

10. J.H.van'tHoff, "Osmotic pressure and chemical equilibrium," Nobel Prize lecture, 13 Dec 1901, http://nobelprize.org/ nobel_prizes/chemistry/laureates/1901/hoff-lecture.pdf.

11. P. C. Hiemenz and T. P. Lodge, Polymer Chemistry: The Basic Concepts, 2nd ed. (CRC Press, 2007).

12. W. Zhang, D. J. Webb, and G.-D. Peng, Proc. SPIE 7753, 77533M (2011).

13. J. Comyn, Polymer Permeability (Elsevier, 1985).

14. F. M. Cox, M. C. J. Large, C. M. B. Cordeiro, R. Lwin, and A. Argyros, Proc. SPIE 7004, 700427 (2007). 